\title{
BASES FOR CUSP FORMS FOR QUASI-FUCHSIAN GROUPS*
}

\author{
IRWIN KRA
}

Let $\Gamma$ be a finitely generated quasi-Fuchsian group of the first kind. Let $\Lambda$ be the limit set of $\Gamma$, and let $\Delta$ and $\Delta^{*}$ be the two invariant components of the region of discontinuity $\Omega$ of $\Gamma$. Denote by $\boldsymbol{A}_{q}(\Delta, \Gamma)$ the space of cusp forms for $\Gamma$ of weight $(-2 q), q \geqq 2$, that are defined on $\Delta$. Let $z_{0} \in \Delta^{*}$ be an ordinary point (that is, not an elliptic fixed point). Let

$$
d=\operatorname{dim} \boldsymbol{A}_{q}(\Delta, \Gamma)=\operatorname{dim} \boldsymbol{A}_{q}\left(\Delta^{*}, \Gamma\right)
$$

and define for $j=0,1,2, \ldots$

$$
\begin{gathered}
f_{j}(z)=\left(z-z_{0}\right)^{-j}, \quad \text { if } \quad z_{0} \in C, \\
f_{j}(z)=z^{-2 q+j}, \quad \text { if } \quad z_{0}=\infty .
\end{gathered}
$$

Let $\varphi_{j}$ be the Poincaré series of $f_{j}$; that is,

$$
\theta f_{j}(z)=\varphi_{j}(z)=\sum_{\gamma \in \Gamma} f_{j}(\gamma z) \gamma^{\prime}(z)^{q}, \quad z \in \Omega,
$$

whenever the series converges uniformly and absolutely on compact subsets of $\Omega$. In this note we shall establish the following

Theorem. The Poincaré series $\varphi_{j}, j=2 q, 2 q+1, \ldots, 2 q+d-1^{6}$ (restricted to $\Delta)$ form a basis for $\boldsymbol{A}_{q}(\Delta, \Gamma)$ if and only if $z_{0}$ is not a Weierstrass point for $\boldsymbol{A}_{q}\left(\Delta^{*}, \Gamma\right)$.

The above theorem is a straightforward application of a construction of Bers [3] of singular Eichler integrals. (See also [1] and [5, Chapter V].) Bases for $A_{q}(\Omega, \Gamma) \cong$ $\boldsymbol{A}_{q}(\Delta, \Gamma) \oplus \boldsymbol{A}_{q}\left(\Delta^{*}, \Gamma\right)$ were investigated in [6], [7], and [4]. It is in general more difficult to obtain explicit bases for $A_{q}(\Delta, \Gamma)$ alone. See in this context the results of Wolpert [9] for Fuchsian groups. A nice feature of the constructions in this paper, is that all the functions considered vary holomorphically with moduli (see Section 3).

* Research supported in part by NSF Grant MCS 8102621. This paper was completed while the author visited the Hebrew University of Jerusalem under a Binational Science Foundation Grant. 
In this paper we use the notation from [6], where many of the topics currently under study are treated in greater detail.

Our main result has as an immediate corollary the following result of Petersson [8]. See also [2].

Theorem. Let $\Gamma$ be a finitely generated Fuchsian group of the first kind acting on the unit disk $\Delta$. Assume that $z=0$ is an ordinary point and not a Weierstrass point for $\boldsymbol{A}_{q}(\Delta, \Gamma)$. Then every element of $\boldsymbol{A}_{q}(\Delta, \Gamma)$ can be written as a Poincaré series of a unique polynomial of degree at most $d-1$.

Proof. It suffices to show that $z=0$ is a Weierstrass point for $A_{q}(\Delta, \Gamma)$ if and only if $z=\infty$ is a Weierstrass point for $\boldsymbol{A}_{q}\left(\Delta^{*}, \Gamma\right)$. Here, of course,

$$
\Delta^{*}=\{z \in C ;|z|>1\} \cup\{\infty\} .
$$

Now every element of $\Gamma$ commutes with the anti-conformal involution

A simple calculation shows that

$$
J: z \mapsto \bar{z}^{-1} .
$$

where for $z \in \Delta^{*}$,

$$
\boldsymbol{A}_{q}(\Delta, \Gamma) \ni \psi \mapsto \psi^{*} \in \boldsymbol{A}_{q}\left(\Delta^{*}, \Gamma\right),
$$

$$
\psi^{*}(z)=\overline{\psi(J z)}\left(\frac{\overline{\partial J}}{\partial \bar{z}}\right)^{q}=(-1)^{q} z^{-2 q} \overline{\psi(J z)},
$$

defines an $\boldsymbol{R}$-linear isomorphism with the property that

$$
\operatorname{ord}_{0} \psi=\operatorname{ord}_{\infty} \psi^{*} \text {. }
$$

This establishes the claim (see Section 1).

\section{Weierstrass points}

Let $\Gamma$ be a non-elementary finitely generated function group with invariant component $\Delta$. Let $\bar{\Delta}$ be the union of $\Delta$ and the parabolic fixed points of $\Gamma$ that represent punctures on $\Delta / \Gamma$. For $z_{0} \in \Delta$, let $v=v\left(z_{0}\right)$ be the order of the stabilizer of $z_{0}$ in $\Gamma$. If $0 \neq \varphi \in A_{q}(\Delta, \Gamma)$, then the order $\mu$ of $\varphi$ at $z_{0}$ satisfies

$$
\mu \geqq 0, \quad \mu+q \equiv 0(\bmod v)
$$

We define the reduced order of $\varphi$ at $z_{0}$ by the formula

$$
\operatorname{red} \operatorname{ord}_{z_{0}} \varphi=\frac{\operatorname{ord}_{z_{0}} \varphi}{v\left(z_{0}\right)} .
$$

Remark. If $z_{0}$ as above is finite, then $\varphi \in A_{q}(\Delta, \Gamma)$ has a Taylor series expansion

$$
\varphi(z)=\sum_{j=\mu}^{\infty} a_{j}\left(z-z_{0}\right)^{j}, \quad a_{\mu} \neq 0,
$$


valid in a neighborhood of $z_{0}$. If $\infty \in \Delta$, then $\varphi \in A_{q}(\Delta, \Gamma)$ satisfies

$$
\varphi(z)=O\left(|z|^{-2 q}\right), \quad z \rightarrow \infty,
$$

and thus the Laurent series of $\varphi$ is of the form

$$
\varphi(z)=\sum_{j=\mu}^{\infty} a_{j} z^{-2 q-j}, \quad a_{\mu} \neq 0,
$$

valid in a neighborhood of $\infty$, and

$$
\mu=\operatorname{ord}_{\infty} \varphi .
$$

As above, all our results and calculations will be invariant under Möbius transformations (compare (0.1) with (0.2)), and by conjugation we may always assume that we are considering one of the following two standard situations:

(I) $0=z_{0} \in \Delta$ and the stabilizer of $z_{0}$ in $\Gamma$ is generated by (here $v \in Z, v \geqq 1$ )

$$
A: \zeta \mapsto e^{2 \pi i / v} \zeta \text {. }
$$

(II) $\infty=z_{0} \in \bar{\Delta} \backslash \Delta$, the stabilizer of $z_{0}$ in $\Gamma$ is generated by

$$
A: \zeta \mapsto \zeta+1 \text {, }
$$

and

$$
U=\{\zeta \in C ; \operatorname{Im} \zeta>0\}
$$

is precisely invariant under $\langle A\rangle$ in $\Gamma$.

Under assumption (II), every $\varphi \in A_{q}(\Delta, \Gamma)$ has a Fourier series expansion

$$
\varphi(\zeta)=\sum_{j=\mu}^{\infty} a_{j} e^{2 \pi i j \zeta}, \quad \zeta \in U,
$$

where $a_{\mu} \neq 0, \mu>0$. We define the order and reduced order of $\varphi$ at $\infty$ by

$$
\operatorname{ord}_{\infty} \varphi=\operatorname{red} \operatorname{ord}_{\infty} \varphi=\mu .
$$

Let $\left(p ; v_{1}, \ldots, v_{n}\right)$ be the signature of $\Gamma$. It was shown in [5, pp. 110-115] that

$$
\sum_{z \in \bar{\Delta} / \Gamma} \operatorname{red~ord}_{z} \varphi=q\left\{2 p-2+\sum_{j=1}^{n}\left(1-\frac{1}{v_{j}}\right)\right\}
$$

for all $\varphi \in A_{q}(\Delta, \Gamma), \varphi \neq 0$.

Let $d=\operatorname{dim} \boldsymbol{A}_{q}(\Delta, \Gamma)$. It is well known (see, for example, [5, pp. 328-330]) that

$$
d=(2 q-1)(p-1)+\sum_{j=1}^{n}\left[q-\frac{q}{v_{j}}\right]
$$

where, as usual, $[x]$ is the greatest integer less than or equal to $x$ and we use the convention $\left[q-\frac{q}{\infty}\right]=\lim _{v \rightarrow \infty}\left[q-\frac{q}{v}\right]=q-1$.

By a basis of $A_{q}(\Delta, \Gamma)$ adapted to $z_{0} \in \bar{\Delta}$, we mean a basis $\left\{\varphi_{1}, \ldots, \varphi_{d}\right\}$ of $A_{q}(\Delta, \Gamma)$ with the property that

$$
\mu_{1}<\mu_{2}<\ldots<\mu_{d},
$$


where

$$
\mu_{j}=\operatorname{ord}_{z_{0}} \varphi_{j}
$$

and if $\infty \neq z_{0} \in \Delta$, then

$$
\varphi_{j}(\zeta)=\left(\zeta-z_{0}\right)^{\mu_{j}}+\sum_{k>\mu_{d}} a_{k}\left(\zeta-z_{0}\right)^{k}, \text { for }\left|\zeta-z_{0}\right| \text { small, }
$$

and if $z_{0}=\infty \in \bar{\Delta} \backslash \Delta$, then

$$
\varphi_{j}(\zeta)=e^{2 \pi i \mu_{j} \zeta}+\sum_{k>\mu_{d}} a_{k} e^{2 \pi i k \zeta}, \quad \zeta \in U
$$

(assuming normalization (II)).

The point $z_{0}$ is a Weierstrass point for $\boldsymbol{A}_{q}(\Delta, \Gamma)$ provided the sequence in (1.3) is not as low as it could possibly be. Thus if $z_{0} \in \Delta$ is an ordinary point (not an elliptic fixed point), then $z_{0}$ is a Weierstrass point if and only if

$$
\mu_{j}>j-1 \text { for some } j=1, \ldots, d \text {. }
$$

If $z_{0} \in \Delta$ is an elliptic fixed point of order $v$, then $z_{0}$ is a Weierstrass point if and only if

$$
\mu_{j}>\mu_{0}+(j-1) v \text { for some } j=1, \ldots, d,
$$

where $\mu_{0}=\mu_{0}\left(z_{0}\right)$ is the smallest non-negative integer of the form $\alpha \nu-q$ with $\alpha \in Z$; that is,

$$
\mu_{0}\left(z_{0}\right)=\mu_{0}=-v\left[-\frac{q}{v}\right]-q
$$

Finally, $z_{0} \in \bar{\Delta} \backslash \Delta$ is a Weierstrass point if and only if

$$
\mu_{j}>j \text { for some } j=1, \ldots, d .
$$

We define the weight of $z_{0}$ by

$$
\begin{gathered}
\tau\left(z_{0}\right)=\sum_{j=1}^{d} \mu_{j}-j+1, \quad \text { if } z_{0} \text { is an ordinary point, } \\
\tau\left(z_{0}\right)=\sum_{j=1}^{d} \mu_{j}-\mu_{0}-j v+v, \quad \text { if } z_{0} \text { is an elliptic fixed point of order } v,
\end{gathered}
$$

and

$$
\tau\left(z_{0}\right)=\sum_{j=1}^{d} \mu_{j}-j, \quad \text { if } z_{0} \text { is a parabolic fixed point. }
$$

It follows that $\tau\left(z_{0}\right) \geqq 0$ and that $\tau\left(z_{0}\right)>0$ if and only if $z_{0}$ is a Weierstrass point.

For any basis $\left\{\varphi_{1}, \ldots, \varphi_{d}\right\}$ of $A_{q}(\Delta, \Gamma)$, we define the Wronskian $W$ of the basis by

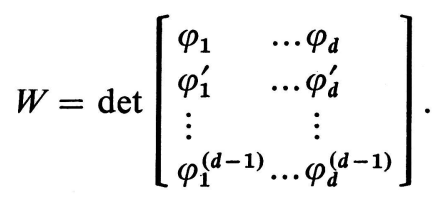


One checks easily that $0 \neq W \in A_{Q}(\Delta, \Gamma)$, where

It follows that

$$
Q=\frac{1}{2} d(2 q-1+d) \text {. }
$$

One shows next that

$$
\sum_{z \in \bar{\Delta} / \Gamma} \operatorname{red~ord}_{z} W=Q\left\{2 p-2+\sum_{j=1}^{n}\left(1-\frac{1}{v_{j}}\right)\right\} .
$$

$$
\operatorname{ord}_{z_{0}} W=\tau\left(z_{0}\right) \text {, for } z_{0} \text { an ordinary point, }
$$

$\operatorname{ord}_{z_{0}} W=\tau\left(z_{0}\right)+d\left(\mu_{0}+(v-1)\left(\frac{d-1}{2}\right)\right)$, for $z_{0}$ an elliptic fixed point of order $v$, and

$$
\operatorname{ord}_{z_{0}} W=\tau\left(z_{0}\right) d+\left(\frac{d+1}{2}\right), \text { for } z_{0} \text { a parabolic fixed point } \in \bar{\Delta} .
$$

We conclude that there are finitely many $\Gamma$-equivalence classes of Weierstrass points.

If $z_{0} \in \Delta$, then for $j \geqq 0$

$$
\boldsymbol{A}_{q}(\Delta, \Gamma) \ni \varphi \stackrel{L_{z_{\mathrm{c}}, j}}{\longrightarrow} a_{j} \in \boldsymbol{C},
$$

with $\varphi$ given by (1.1), is a linear functional. There are two reasons why this linear functional might be the zero linear functional. First, it might be zero because

$$
j+q \neq 0(\bmod v)
$$

or because the $j$-th coefficient of the Taylor series of every $\varphi \in A_{q}(\Delta, \Gamma)$ vanishes, even though

$$
j+q \equiv 0(\bmod v) .
$$

If $\varphi_{1}, \ldots, \varphi_{d}$ is a basis for $\boldsymbol{A}_{q}(\Delta, \Gamma)$ adapted to $z_{0}$ and (1.4) holds, then

$$
L_{z_{0}, \mu_{j}}\left(\varphi_{k}\right)=\delta_{j k}, \quad 1 \leqq j, \quad k \leqq d ;
$$

thus the linear functionals

$$
L_{z_{0}, \mu_{1}}, \ldots, L_{z_{0}, \mu_{d}}
$$

form a basis for the dual space $\boldsymbol{A}_{q}(\Delta, \Gamma)^{*}$ of $\boldsymbol{A}_{q}(\Delta, \Gamma)$.

A similar analysis can be carried over to cusps using (1.2). It is important to note that the linear functionals $L_{z_{0}, j}$ extend to the space of meromorphic automorphic forms on $\Delta$ that are regular at $z_{0}$.

Remark. Let $\Gamma$ be a non-elementary finitely generated Kleinian group with region of discontinuity $\Omega$. Let $\Delta_{1}, \ldots, \Delta_{r}$ be a maximal set of inequivalent components of $\Omega$. Let $\Gamma_{j}$ be the stabilizer of $\Delta_{j}$. Then

$$
\boldsymbol{A}_{q}(\Omega, \Gamma)=\bigoplus_{j=1}^{r} \boldsymbol{A}_{q}\left(\Delta_{j}, \Gamma_{j}\right)
$$

Thus the above analysis may be extended to the space of all cusp forms for $\Gamma$. 


\section{Singular Eichler integrals}

Let $\Gamma$ be a non-elementary finitely generated Kleinian group with region of discontinuity $\Omega$ and limit set $\Lambda$. Let $q \in Z, q \geqq 2$. Choose once and for all $2 q-1$ distinct points $a_{1}, \ldots, a_{2 q-1}$ in $\Lambda$. Define a meromorphic function $f(z, \zeta)$ on $\Omega \times \Omega$ by the formula

$$
f(z, \zeta)=-\frac{1}{2 \pi} \frac{1}{\zeta-z} \prod_{j=1}^{2 q-1} \frac{z-a_{j}}{\zeta-a_{j}}
$$

The definitions of various terms and the conventions regarding $z, \zeta$ or $a_{j}=\infty$ are those of [6]. We also define

$$
\varphi(z, \zeta)=\sum_{\gamma \in \Gamma} f(z, \gamma \zeta) \gamma^{\prime}(\zeta)^{q} .
$$

The series in (2.1) converges uniformly and absolutely on compact subsets of

$$
\{(z, \zeta) \in \Omega \times \Omega ; z \neq \gamma \zeta, \quad \text { all } \gamma \in \Gamma\} .
$$

For fixed $b \in \Omega, \varphi(b, \cdot)$ is the Poincaré series of $f(b, \cdot)$ :

$$
\varphi(b, \cdot)=\theta f(b, \cdot) .
$$

Thus, $\varphi(b, \cdot)$ is a meromorphic automorphic form of weight $(-2 q)$ that satisfies the cusp condition (at each cusp in $\Omega$ ). The function $\varphi(b, \cdot)$ has a simple pole at $b$ (and its images under $\Gamma$ ) whenever

$$
q \equiv 1(\bmod v(b))
$$

and is regular at $b$ (thus a cusp form; that is, an element of $\boldsymbol{A}_{q}(\Omega, \Gamma)$ ) otherwise. For fixed $b \in \Omega, \varphi(\cdot, b)$ is a meromorphic Eichler integral. This integral is parabolic and is regular at the cusps. The integral has a simple pole at $b$ whenever

$$
q \equiv 0(\bmod v(b))
$$

and is regular at $b$ (thus in $E_{1-q}^{b}(\Omega, \Gamma)$ ) otherwise. In the latter case, $\varphi(\cdot, b)$ is identically zero. Furthermore, for $m \in Z, m \geqq 1$, we define

$$
\varphi_{m}(z, \zeta)=\frac{\partial^{m-1} \varphi(z, \zeta)}{\partial \zeta^{m-1}}
$$

One checks easily that $\varphi_{m}(\cdot, b)$ is a meromorphic Eichler integral with a singularity at $b$ of the form

$$
\frac{(-1)^{m-1}}{2 \pi}(m-1) ! v(b)(z-b)^{-m}
$$

whenever

$$
m \equiv 1-q(\bmod v(b))
$$


and $\varphi_{m}(\cdot, b)$ is identically zero otherwise. Using a partial fraction decomposition one sees that

$$
\psi_{m}(z, \zeta)=\frac{\partial^{2 q-1} \varphi_{m}(z, \zeta)}{\partial z^{2 q-1}}=\frac{\partial^{2 q+m-2} \varphi(z, \zeta)}{\partial z^{2 q-1} \partial \zeta^{m-1}}
$$

defines a function on $\Omega \times \Omega$ that is given by the formula

$$
\begin{gathered}
\psi_{m}(z, \zeta)=-\frac{(2 q-1) !}{2 \pi} \frac{\partial^{m-1}}{\partial \zeta^{m-1}} \sum_{\gamma \in \Gamma} \frac{\gamma^{\prime}(\zeta)^{q}}{(\gamma \zeta-z)^{2 q}} \\
=-\frac{(2 q-1) !}{2 \pi} \frac{\partial^{m-1}}{\partial \zeta^{m-1}} \sum_{\gamma \in \Gamma} \frac{\gamma^{\prime}(\zeta)^{q}}{\left(\gamma \zeta-\gamma \circ \gamma^{-1} z\right)^{2 q}} \\
=-\frac{(2 q-1) !}{2 \pi} \frac{\partial^{m-1}}{\partial \zeta^{m-1}} \sum_{\gamma \in \Gamma} \frac{\gamma^{\prime}(\zeta)^{q}}{\left(\zeta-\gamma^{-1} z\right)^{2 q} \gamma^{\prime}(\zeta)^{q} \gamma^{\prime}\left(\gamma^{-1} z\right)^{q}} \\
=-\frac{(2 q-1) !}{2 \pi} \frac{\partial^{m-1}}{\partial \zeta^{m-1}} \sum_{\gamma \in \Gamma} \frac{\left(\gamma^{-1}\right)^{\prime}(z)^{q}}{\left(\zeta-\gamma^{-1} z\right)^{2 q}}=-\frac{(2 q-1) !}{2 \pi} \frac{\partial^{m-1}}{\partial \zeta^{m-1}} \sum_{\gamma \in \Gamma} \frac{\gamma^{\prime}(z)^{q}}{(\zeta-\gamma z)^{2 q}} \\
=(-1)^{m} \frac{(2 q+m-2) !}{2 \pi} \sum_{\gamma \in \Gamma} \frac{\gamma^{\prime}(z)^{q}}{(\zeta-\gamma z)^{2 q+m-1}} .
\end{gathered}
$$

It follows that for fixed $b \in \Omega, \psi_{m}(\cdot, b)$ is a meromorphic automorphic form that satisfies the cusp condition at each cusp. Its singularity at $b$ is of the form

$$
(-1)^{m} \frac{(2 q+m-2) !}{2 \pi} v(b)(b-z)^{2 q+m-1},
$$

whenever $m$ satisfies (2.2), and is the zero form otherwise.

We let $\boldsymbol{E}_{1-q}^{\mathrm{mer}}(\Omega, \Gamma)$ denote the linear span of the Eichler integrals

$$
\varphi_{m}(\cdot, b), \quad b \in \Omega, m=1,2,3, \ldots
$$

Thus every $\varphi \in \boldsymbol{E}_{1-q}^{\mathrm{mer}}(\Omega, \Gamma)$ is regular on a fundamental set $\omega$ for $\Gamma$ on $\Omega$ except for finitely many singularities. Such a $\varphi$ induces a linear functional $\varphi^{*}$ on $\boldsymbol{A}_{q}(\Omega, \Gamma)$ by the formula

$$
\varphi^{*}(\psi)=\sum_{b \in \omega} \frac{\operatorname{res}_{b} \varphi \psi}{v(b)}, \quad \varphi \in \boldsymbol{E}_{1-q}^{\mathrm{mer}}(\Omega, \Gamma), \quad \psi \in \boldsymbol{A}_{q}(\Omega, \Gamma) .
$$

A basic theorem of Bers [3] (see also Ahlfors [1] and Kra [5, Chapter V]) asserts that $\varphi$ is the restriction to $\Omega$ of a polynomial of degree $\leqq 2 q-1$ if and only if $\varphi^{*}$ is the zero linear functional.

Theorem. Let $\Gamma$ be a finitely generated quasi-Fuchsian group of the first kind. Let $\Delta$ and $\Delta^{*}$ be the two invariant components of $\Gamma$. Let $d=\operatorname{dim} A_{q}(\Delta, \Gamma), q \geqq 2$.

(a) Let $b_{1}, \ldots, b_{d}$ be $d$ distinct points of $\Delta^{*}$ with the property that every $\varphi \in \boldsymbol{A}_{q}\left(\Delta^{*}, \Gamma\right)$ that vanishes at $b_{j}, j=1, \ldots, d$, must be identically zero. Then the restric- 
tions of the functions

$$
\psi_{1}\left(\cdot, b_{1}\right), \ldots, \psi_{1}\left(\cdot, b_{d}\right)
$$

to $\Delta$ form a basis for $\boldsymbol{A}_{q}(\Delta, \Gamma)$.

(b) Let $b \in \Delta^{*}$. Let $\mu_{1}<\mu_{2}<\ldots<\mu_{d}$ be the orders of vanishing at $b$ for cusp forms in $\boldsymbol{A}_{q}\left(\Delta^{*}, \Gamma\right)$. Then the restrictions of the functions

$$
\psi_{\mu_{j}+1}(\cdot, b), \quad j=1, \ldots, d,
$$

to $\Delta$ form a basis for $\boldsymbol{A}_{q}(\Delta, \Gamma)$.

Proof. The hypotheses imply that the Eichler integrals

$$
\begin{gathered}
\varphi_{1}\left(\cdot, b_{1}\right), \ldots, \varphi_{1}\left(\cdot, b_{d}\right), \quad(\operatorname{case}(a)) \\
\varphi_{\mu_{1}+1}(\cdot, b), \ldots, \varphi_{\mu_{d}+1}(\cdot, b),(\operatorname{case}(b))
\end{gathered}
$$

are linearly independent modulo polynomials of degree $2 q-2$ even when restricted to $\Delta$. It follows that their $(2 q-1)$-derivatives restricted to $\Delta$ are linearly independent.

Remarks. (1) The above theorem generalizes the theorem in the introduction.

(2) For $q=2$, part (a) of the above theorem was obtained independently by Clifford Earle using different methods (private communication).

(3) Bers [3] has also constructed Eichler integrals with prescribed singularities at cusps. These constructions are not as explicit as the ones involving singularities only at points of $\Omega$.

(4) The linear functionals on $\boldsymbol{A}_{q}(\Omega, \Gamma)$

$$
\varphi_{m}^{*}(\cdot, b), b \in \Omega,
$$

are, of course, constant multiples of the maps $L_{b, m}$ discussed in Section 1.

\section{Spaces of cusp forms over Teichmüller spaces}

Let $\Gamma$ be a finitely generated Fuchsian group of the first kind acting on the upper half plane $U$. Let $M(\Gamma)$ be the space of Beltrami coefficients for $\Gamma$ supported on $U$. For $\sigma \in M(\Gamma)$, let $w^{\sigma}$ be the unique normalized (fixing $0,1, \infty$ ) homeomorphism of $\boldsymbol{C} \cup\{\infty\}$ that is $\sigma$-conformal in $U$ and conformal in the lower half plane $U^{*}$. Two Beltrami coefficients $\sigma_{1}$ and $\sigma_{2}$ are equivalent if $w^{\sigma_{1}}\left|U^{*}=w^{\sigma_{2}}\right| U^{*}$. The set of equivalence classes $[\sigma]$ of Beltrami coefficients $\sigma$ forms the Teichmüller space $T(\Gamma)$. The Bers fiber space $F(\Gamma)$ is defined as

$$
F(\Gamma)=\left\{([\sigma], z) ;[\sigma] \in T(\Gamma), z \in w^{\sigma}(U)\right\} .
$$

For fixed $b \in U^{*}$, the function

$$
T(\Gamma) \ni[\sigma] \mapsto w^{\sigma}(b) \in C \backslash\{0,1\}
$$


is holomorphic. Furthermore, since $w^{\sigma} \mid U^{*}$ induces a conformal map between $U^{*} / \Gamma$ and $w^{\sigma}\left(U^{*}\right) / w^{\sigma} \Gamma\left(w^{\sigma}\right)^{-1}$, it follows that $b$ is a Weierstrass point for $\boldsymbol{A}_{q}\left(U^{*}, \Gamma\right)$ if and only if $w^{\sigma}(b)$ is a Weierstrass point for $A_{q}\left(w^{\sigma}\left(U^{*}\right), w^{\sigma} \Gamma\left(w^{\sigma}\right)^{-1}\right)$. The results of the previous section imply the following

Theorem. Let $b_{1}, \ldots, b_{k}$ be $k$ distinct points in $U^{*}$. For each $j, j=1, \ldots, k$, let

$$
0<\mu_{j 1}<\mu_{j 2}<\ldots<\mu_{j m_{j}}
$$

be $m_{j} \geqq 1$ positive integers. Then

$$
f_{j i}([\sigma], z)=\left(z-w^{\sigma}\left(b_{j}\right)\right)^{-2 q+1-\mu_{j i},} \quad 1 \leqq i \leqq m_{j}, \quad 1 \leqq j \leqq k,
$$

are holomorphic functions on $F(\Gamma)$, and we set

$$
\psi_{j i}([\sigma], z)=\sum_{\gamma \in \Gamma} f_{j i}\left([\sigma], \gamma^{\sigma} z\right)\left(\gamma^{\sigma}\right)^{\prime}(z)^{q},
$$

where $i$ and $j$ are as above, and

$$
\gamma^{\sigma}=w^{\sigma} \circ \gamma \circ\left(w^{\sigma}\right)^{-1} \text { for } \quad \gamma \in \Gamma .
$$

Let $\mathscr{R}$ be the linear span of the $f_{j i}$ and $\boldsymbol{A}$ the linear span at the $\psi_{j i}$. Then $\mathscr{R}$ is a trivial vector bundle over $T(\Gamma)$ of rank $\sum_{j=1}^{k} m_{j}$ and $\boldsymbol{A}$ is a trivial vector bundle over $T(\Gamma)$ of rank $r$, where $r$ is the rank of the matrix

$$
\begin{gathered}
\left(\tilde{f}_{k}^{*}\left(\varphi_{l}\right)\right)_{l=1, \ldots, \operatorname{dim} A_{q}\left(U^{*}, \Gamma\right),} \\
k \text { ranges over }(j, i) \text { as above, }
\end{gathered}
$$

the $\left\{\varphi_{l} ; l=1, \ldots, \operatorname{dim} \boldsymbol{A}_{q}\left(U^{*}, \Gamma\right)\right\}$ form a basis for $\boldsymbol{A}_{q}\left(U^{*}, \Gamma\right)$, and $\tilde{f}_{j i}$ is the Eichler integral constructed in Section 2 whose only singularity is a pole of order $\mu_{j i}$ at $b_{j}$ (and equivalent points) with principal part

provided

$$
\left(z-b_{j}\right)^{-\mu_{j i}}
$$

and $\tilde{f}_{j i}=0$, otherwise.

$$
\mu_{j i} \equiv 1-q\left(\bmod v\left(b_{j}\right)\right)
$$

Furthermore, the Poincaré series operator $\theta$ is a vector bundle map $\theta: \mathscr{R} \rightarrow \boldsymbol{A}$ and the kernel of $\theta$ is a trivial vector bundle over $T(\Gamma)$ of rank $\sum_{j=1}^{m} m_{j}-r$.

Proof. The proof of the above theorem is quite similar to the proof of Theorem 11.2 of [6].

\section{References}

[1] AhLfors, L. V.: The structure of a finitely generated Kleinian group. - Acta Math. 122, 1969, $1-17$.

[2] Bers, L.: Completeness theorems for Poincaré series in one variable. - Proceedings of the International Symposium on Linear Spaces. Jerusalem 1960, Jerusalem, 1961. 88-100.

[3] Bers, L.: Eichler integrals with singularities. - Acta Math. 127, 1971, 11-22. 
[4] Hejhal, D. A.: Monodromy groups and Poincaré series. - Bull. Amer. Math. Soc. 84, 1978, $339-376$.

[5] KRA, I.: Automorphic forms and Kleinian groups. - Benjamin, Reading, Massachusetts, 1972.

[6] KRA, I.: On the vanishing of and spanning sets for Poincaré series for cusp forms. - Acta Math. $153,1984,47-116$.

[7] KRA, I., and B. MASKIT: Bases for quadratic differentials. - Comment. Math. Helv. 57, 1982, $603-626$.

[8] Petersson, H.: Über Weierstrasspunkte und die expliziten Darstellungen der automorphen Formen von reeller Dimension. - Math. Z. 52, 1949, 32-59.

[9] Wolpert, S.: The Fenchel-Nielsen deformation. - Ann. of Math. (2) 115, 1982, 501-528.

State University of New York at Stony Brook

Department of Mathematics

Stony Brook, New York 11794

USA

Received 21 November 1983 